\title{
Hollow-cone Foucault Imaging of Magnetic Microstructures in Large Magnetostrictive FeGa Alloy
}

Shigeo Mori ${ }^{1}$, A Kawaguchi ${ }^{1}$ and Ken $\operatorname{Harada}^{2}$

${ }^{1}$ Osaka Prefecture University, Sakai, Osaka, Japan, ${ }^{2}$ RIKEN, Hatoyama, Saitama, Japan

Magnetostrictive materials exhibit external form changes by applying an external magnetic field. Recently, FeGa alloys has attracted much attention as a practical magnetostrictive material because they exhibit a large magnetostriction at low magnetic field [1]. The magnetostriction of FeGa alloy should originate from the reorientation of magnetoelastically and magnetostatically autarkic rigid micro-'cells', and thus, dynamical behaviors of the magnetic domain structures by applying external magnetic fields should have strong relationship with the large magnetostriction in FeGa alloys. In this study, we have investigated the magnetic microstructures of large magnetostrictive FeGa alloys by using Lorentz microscopy, Hollow-Cone Foucault imaging, and small-angle electron diffraction (SmAED) techniques [2].

Recently, we developed Hollow-Cone Foucault (HCF) imaging method as Lorentz microscopy, where an incident electron beam on the specimen was tilted with respect to the optical axis with an inclination angle and was circulated in all azimuths around the optical axis [3]. In the HCF dark-field image, both magnetic domains and magnetic domain walls were simultaneously visualized under the infocus condition. The objective lens is switched off to obtain a magnetic field-free space around the specimen and the objective mini-lens is used to focus a crossover just on the selected area (SA) aperture. The SA aperture is used as an angle limiting tool. The parallel electron beams having less than $10^{-6}$ rad diffusion angle are irradiated on the specimen region of about $85 \mathrm{~mm}$ in diameter. The circulating electron beam is illuminated in all azimuthal directions around the optical axis. Magnetic domain walls are observed with black lines and several domains with a slight bright contrast. Figure 1(a) shows a typical dark-field HCF image for 5.0 $\times 10^{-}$ 4 rad inclination angles. The SA aperture with hole size of $100 \mathrm{~mm}$ in diameter corresponding to the $1.30 \times 10^{-3}$ rad was utilized as the angle-limiting tool. Magnetic domain walls with dark lines are observed, in addition to several domains having slight bright contrast. With the help of SmAED and Foucault imaging experiments, we determined spatial distribution of magnetization in each magnetic domains, as schematically illustrated in Figure 1(b).

Figure 2 shows magnetic microstructures obtained using Fresnel method in the (001) plane of the FeGa thin film without external magnetic fields. In the Fresnel image, bright and dark lines identified as magnetic domain walls can be clearly seen. In Figure 2, yellow arrows indicate the direction of the magnetizations in each magnetic domains. The $180^{\circ}$ and $90^{\circ}$ magnetic domains are formed at room temperature without external magnetic field. Note that magnetic moments are oriented along the [100] direction. When a magnetic field of $400 \mathrm{Oe}$ is applied perpendicular to the film, the magnetic moments should rotate in the in-plane direction toward the [010] direction and the magnetic domains with magnetization along the [100] direction are dominant at the expense of magnetic domains with the magnetic moments along the [001] direction. In this talk, we will report dynamics of the magnetic domains under the vertical magnetic field. 


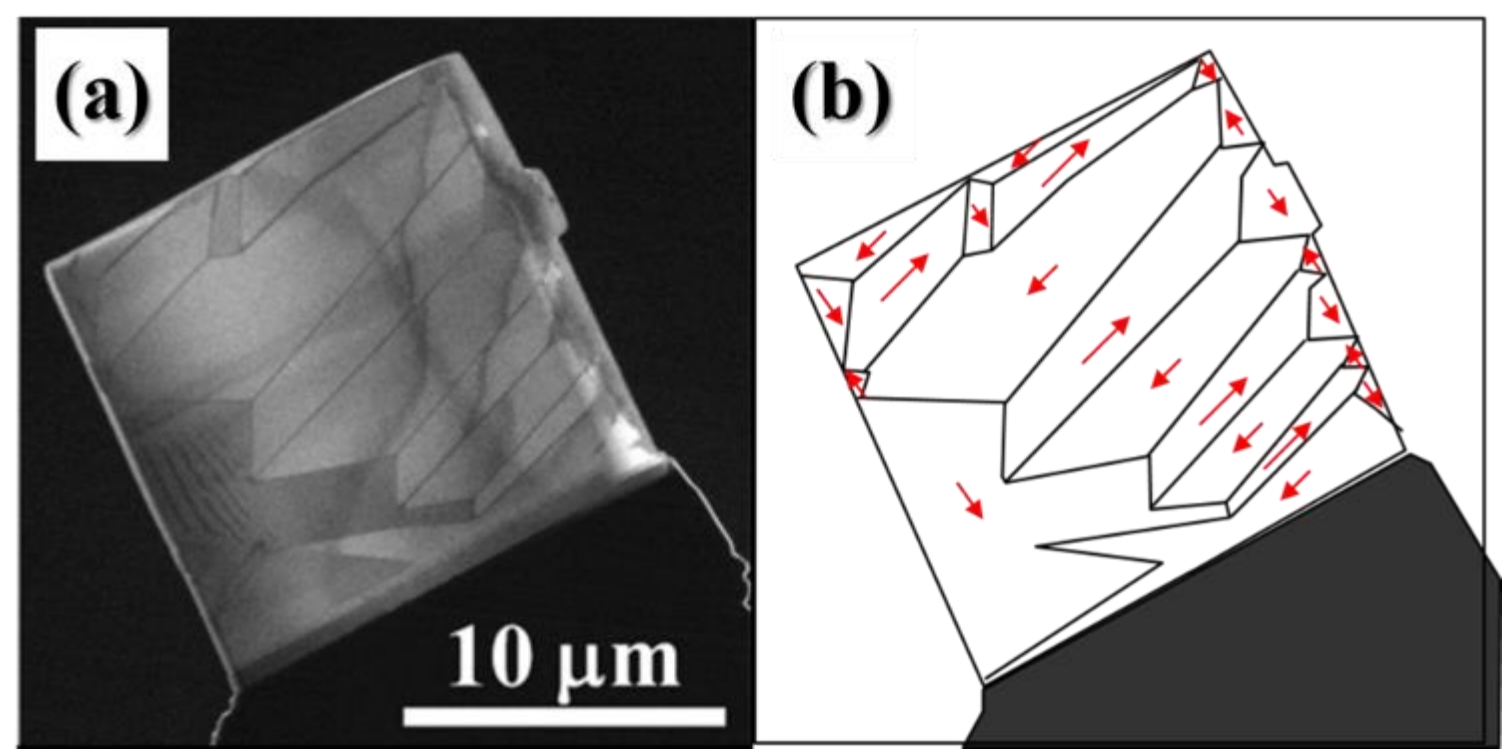

Figure 1. (a) Dark-field hollow-cone Foucault image. (b) Schematic description of magnetic domain structure of (a). Red arrows indicate the direction of magnetic moments in each domains.

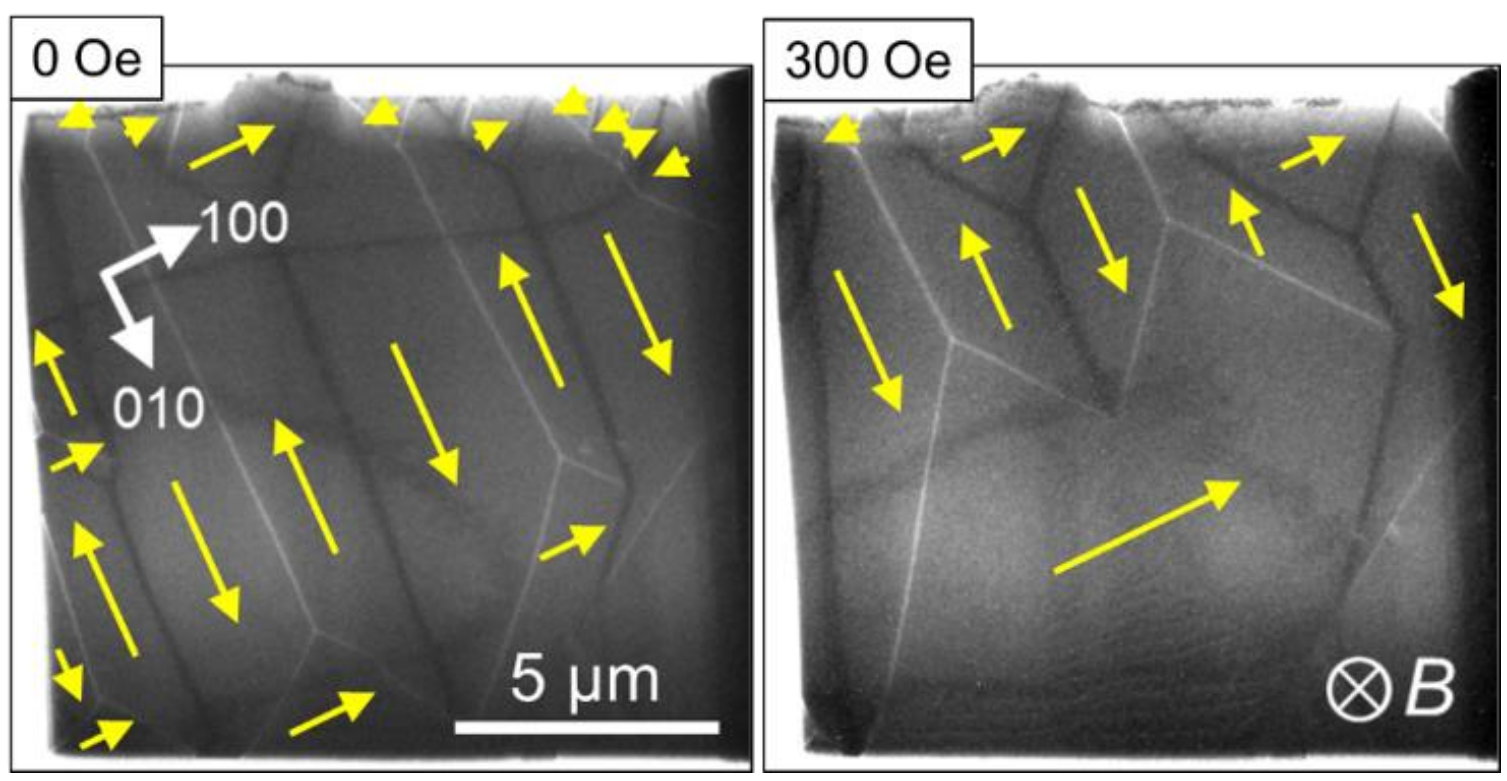

Figure 2. Changes of magnetic domain structures by applying the strength of magnetic fields of (a) 0 Oe and (b) 300 Oe.

\section{References}

[1] C. Harsh, et al., Nature 521, 340-343 (2015).

[2] H. Nakajima et al., Microscopy, 67, 207-213 (2018).

[3] K. Harada et al., Applied Physics Express 12, 042003 1-4 (2019). 